\title{
The confidence of school's nutrition project: urinary analysis
}

\author{
A. Nishide ${ }^{1}$, M. Yoshioka ${ }^{2}$, A. Hata ${ }^{1}$ and S. Sato ${ }^{2,3}$ \\ ${ }^{1}$ Department of Public health, Chiba University, 1-8-1 Inohana, Chuo-ku, Chiba-shi, Chiba-ken, Japan, ${ }^{2}$ Chiba \\ Prefectural Institute of Public Health, 666-2 Nitona-cho, Chuo-ku, Chiba-shi, Chiba-ken, Japan and Institute of \\ Public Nutrition Practice,Osaka Prefectre University,1-1 Gakuen-cho, Naka-ku, Sakai-Shi, Osaka, Japan
}

Japan still has been concerned about having a salt intake that is more than the recommended value worldwide ${ }^{(1-3)}$, although the $\mathrm{WHO}^{(4)}$ published new guidelines for recommended intake levels of sodium for adults and children. Particularly, they are concerned that the average salt intake in Japan, an eastern Asian country, is higher than other developed countries ${ }^{(2,3)}$, even though , according to the National Health and Nutrition Survey in Japan ${ }^{(5)}$, salt intake has been continuously decreasing because of the westernisation of the Japanese diet and the use of home refrigerators, instead of salt-preserving. However, the result of a previous study shows that younger Japanese consume almost the same amount of sodium as older people. The authors concluded that people cannot be expected to voluntarily reduce salt intake without encouragement. Another previous study ${ }^{(7)}$ shows a significant effect of the nutrition project towards salt intake reduction among children by urinary analysis. The school program for healthy eating could be an excellent method for maintaining the Japanese diet. The aim of the present study was to assess the effect of the school's nutrition project on salt intake reduction by the analysis of overnight urine.

First, we conducted 3 varieties of nutrition projects ${ }^{(7)}$ to encourage 3 classes and to set 2 classes as a control at Year 4 in 2 public elementary schools in Choshi city. We checked the change of sodium concentrate in the overnight urine specimens collected before and after the intervention, in order to investigate the effect of the Food Education Program. The value of salt intake was estimated from the sodium and creatinine concentration in the overnight urine.

\begin{tabular}{|c|c|c|c|c|c|c|c|c|c|}
\hline group & $\mathrm{n}$ & pre $(0 w)$ mean & SD & post(3w)Mean & SD & follow up (13w) Mean & SD & $\begin{array}{l}\mathrm{p} \text { value for } \\
\text { pre vs. post }\end{array}$ & $\begin{array}{l}\mathrm{p} \text { value for } \\
\text { pre vs. follow up }\end{array}$ \\
\hline intervention & 55 & 5.48 & $3 \cdot 78$ & 4.83 & $2 \cdot 36$ & 4.73 & $2 \cdot 91$ & 0.031 & $0 \cdot 026$ \\
\hline control & 28 & $5 \cdot 24$ & 3.06 & $5 \cdot 18$ & $2 \cdot 86$ & $4 \cdot 71$ & 3.05 & 0.904 & $0 \cdot 355$ \\
\hline
\end{tabular}

(Repeated ANCOVA, followed by fisher's LSD (Salt intake 0w as a covariates))

When compared with the mean of estimated salt intake at pre $(0 \mathrm{w})$ in the intervention group, significant differences were found $(\mathrm{p}<$ $0.05)$ in both the means at post ( $3 w)$ and follow up (13w). On the other hand, no significant difference $(p>0.05)$ was found among the means of estimated salt intake over the 3 intervals $(0 \mathrm{w}, 3 \mathrm{w}, 13 \mathrm{w})$ in urine measurement in the control group. This result suggests that the school's nutrition project might have a benefit on reducing the salt intake among Year 4 pupils. The enhancement and improvement of school's nutrition projects are needed for its efficacy to be made.

1. Intersalt Cooperative Research Group (1988) BMJ 297, 319-328.

2. Stamler J, Elliott P, Dennis B et al. (2003) J Hum Hypertens 17, 591-608.

3. Zhou BF, Stamler J, Dennis B et al. (2003) J Hum Hypertens 17, 623-630.

4. World Health Organization (2012) Guideline: Sodium Intake for Adults and Children. Geneva: WHO.

5. Cancer Control and Health Promotion Division, HealthService Bureau, Ministry of Health Labour and Welfare,Japan,(2011)The National Health and Nutrition Survey in Japan. http://www.mhlw.go.jp/bunya/kenkou/eiyou/dl/h23-houkoku.pdf (accessed October 2013) (in Japanese).

6. Asakura K, Uechi K, Sasaki Y et al. (2014) Br J Nutr 112(7):1195-1205.

7. Mori M, Mori H, Yamori Y (2011) Procedia Social Behavioral Sciences 15:3784-3791. 